\title{
Article \\ High-Voltage Cable Condition Assessment Method Based on Multi-Source Data Analysis
}

\author{
Xiao-Kai Meng ${ }^{1,2}$, Yan-Bing Jia ${ }^{1}$, Zhi-Heng Liu ${ }^{3,4, *}$, Zhi-Qiang Yu ${ }^{3}$, Pei-Jie Han ${ }^{5}$, Zhu-Mao Lu ${ }^{2}$ and Tao Jin ${ }^{2}$ \\ 1 College of Electrical and Power Engineering, Taiyuan University of Technology, Taiyuan 032100, China; \\ mengxiaokai@sx.sgcc.com.cn (X.-K.M.); jiayanbing@tyut.edu.cn (Y.-B.J.) \\ 2 State Grid Shanxi Electric Power Research Institute, Taiyuan 032100, China; \\ luzhumao@sx.sgcc.com.cn (Z.-M.L.); jintao@sx.sgcc.com.cn (T.J.) \\ 3 College of Electronic Information Engineering, Hebei University, Baoding 071002, China; \\ yuzhiqiang@stumail.hbu.edu.cn \\ 4 Key Laboratory of the Ministry of Education on Optoelectronic Information Technology, Tianjin University, \\ Tianjin 300072, China \\ 5 State Grid Shanxi Electric Power Corporation, Taiyuan 032100, China; bhshanpeijie@sx.sgcc.com.cn \\ * Correspondence: liuzhiheng@hbu.edu.cn
}

check for updates

Citation: Meng, X.-K.; Jia, Y.-B.; Liu, Z.-H.; Yu, Z.-Q.; Han, P.-J.; Lu, Z.-M.; Jin, T. High-Voltage Cable Condition Assessment Method Based on Multi-Source Data Analysis. Energies 2022, 15, 1369. https://doi.org/ $10.3390 /$ en15041369

Academic Editor: Andreas Sumper

Received: 4 January 2022

Accepted: 11 February 2022

Published: 14 February 2022

Publisher's Note: MDPI stays neutral with regard to jurisdictional claims in published maps and institutional affiliations.

Copyright: (c) 2022 by the authors. Licensee MDPI, Basel, Switzerland. This article is an open access article distributed under the terms and conditions of the Creative Commons Attribution (CC BY) license (https:// creativecommons.org/licenses/by/ $4.0 /)$.

\begin{abstract}
In view of the problem that the weight value given by the previous state evaluation method is fixed and single and cannot analyze the influence of the weight vector deviation on the evaluation result, a method based on the weight space Markov chain and Monte Carlo method (Markov chains Monte Carlo, MCMC) is proposed. The sampling method is used for evaluating the condition of high-voltage cables. The weight vector set obtained by MCMC sampling and the comprehensive degradation degree of the high-voltage cable sample are weighted and summed then compared in pairs to obtain the comprehensive degradation degree result. The status probability value and overall priority ranking probability of the object to be evaluated are obtained based on probability statistics, and the order of maintenance is determined according to the status probability value and the ranking result. It is realized that the cable line that needs to be identified in the follow-up defect is clarified according to the evaluation result. This is helpful for operational and maintenance personnel to more accurately implement the maintenance plan for the cable and improve the operational and maintenance efficiency.
\end{abstract}

Keywords: high voltage cable; Markov chain Monte Carlo; state evaluation; weight space

\section{Introduction}

We have made great progress in high-level voltage and large-capacity power transmission with XLPE cables in recent years. High-voltage cables of $110 \mathrm{kV}$ and above have been widely used in cities in China [1]. Factors such as the operating environment, working conditions and external forces of the cable may cause defects in operation. Nowadays, a variety of online monitoring systems for cable temperature, grounding circulation, partial discharge, etc. have been commonly installed on high-voltage cables of $110 \mathrm{kV}$ and above [2]. However, the lack of integration of each cable monitoring system makes the monitoring data of various status variables relatively independent and unable to be comprehensively utilized.

Domestic and foreign research results on power cables are mainly focused on analyzing and judging the operating status of power cables, such as on-line monitoring of cable temperature [3], grounding circulation and partial discharge [4], on-line insulation measurement and infrared thermal imaging technology [5], etc. The methods are relatively simple for accurately judging the operating status of the cable, and they have not formed a comprehensive and systematic power cable operating status evaluation model. The research on the identification of high-voltage cable defects mainly focuses on the identification of partial discharge patterns (creeping discharge and corona discharge, etc.) [6], 
using the detection signal changes caused by cable defects (partial discharge signals) and directly using the detection signal threshold for fault warning and judgement [7]. There are many other types of defects in cables, except for partial discharge in actual situations, such as abnormal cable joint temperature and outer sheath damage [8]. Therefore, the cable-related index data obtained from the offline inspection of the cable and the online inspection can reflect the insulation condition of the cable to a certain extent. However, the research methods are relatively simple, and the multi-source information of the cable is not comprehensively considered, so it is impossible to establish a systematic cable condition evaluation model to comprehensively evaluate the cable condition.

Nowadays, scholars have begun to study comprehensive state evaluation methods of cables and have achieved some results. This has established a $10 \mathrm{kV}$ cable operating state evaluation model based on the test results obtained by conducting macroscopic and microscopic tests on the test cable, and has fully considered the cable operating life, operating environment and other ancillary factors, and evaluated the new and old cables $[9,10]$. It applies D-S evidence theory to the state assessment of cables [11], uses multiple monitoring indicators, and proposes a comprehensive evaluation model for cable insulation that integrates evidence theory and fuzzy theory. It also established a comprehensive indicator system for cables [12], and obtained the weight values of various indicators based on the improved analytic hierarchy process [13]. On this basis, a comprehensive evaluation model of cable operating status with improved radar chart theory was established [14]. An analysis method combining fuzzy theory and analytic hierarchy process has been introduced to evaluate the cable status based on the particularity and difference of different influencing factors [15], and on the basis of the evaluation results, a maintenance strategy considering economy and reliability is formulated [16]. The insulation resistance, dielectric loss angle and partial discharge of the disabled cables and their accessories were comprehensively measured. A cable condition assessment model was established based on the measurement results and the national standard GB12706 [17]. Compared with the cable body, the terminals and intermediate joints of the cable accessories are more prone to failure due to their complicated design and production defects $[18,19]$. The aforementioned cable state evaluation methods all give fixed weight values, but do not consider the difference and uncertainty of the group evaluation weight vector. It is impossible to analyze the influence of the weight value on the evaluation result when the weight value changes within a given interval, nor can it give the probability value of each state of the object to be evaluated. A thermal state evaluation method based on the maximum temperature difference on the terminal surface is proposed by analyzing the thermal field distribution characteristics of the test cable terminal surface [20]. The multi-state index evaluation of high-voltage cables is a multi-attribute comprehensive evaluation problem, and the weight of each index has a great impact on the evaluation result [21]. The advantages of Markov chain Monte Carlo (MCMC) in the evaluation of multi-state indicators are followed, which can solve the problem of the influence of the weights of different indicators on the evaluation result. MCMC requires to evaluate the full data likelihood at different parameter values iteratively [22]. MCMC users often decide convergence by applying empirical diagnostic tools in practice [23]. It is applied to multi-source data analysis of cable PD ultrasonic signals based on the engineering value of MCMC [24]. The high-voltage cables work in a very strong electric field, and the existing online and offline detection technologies, test methods, condition evaluation methods, and defect identification methods are not complete. How to comprehensively utilize and quantify the multi-source data of cables as the basis for evaluating the status of high-voltage cables is a difficult problem for improving the reliability and safety of the power supply operation system.

In order to solve the problem that the weight value of the high-voltage cable status evaluation is fixed and single and cannot analyze the influence of the weight deviation on the evaluation result; the cable status evaluation system is established based on the weight space of MCMC sampling, and the multi-source status indicator system of the cable is built. 
The proposed method was verified by an example based on the relevant data provided by a certain electric power bureau.

\section{The Weight Space of Multi-Attribute Comprehensive Evaluation}

In order to fully dig the multi-source data such as online monitoring and offline testing of high-voltage cables, comprehensive evaluation of the operating status of high-voltage cables is carried out in consideration of the difference and uncertainty of the weight value. In actual situations, the weight samples determined by a small number of expert groups are often limited, and the comprehensive evaluation results obtained with only a few weight vectors are not necessarily accurate, and it is impossible to analyze the influence of the weight vector deviation on the comprehensive evaluation results. According to the limited weight vector data, the random distribution that the weight vector of the expert group may satisfy is estimated, and the amount of available weight sample data is artificially expanded, which can make the evaluation result more reasonable.

\subsection{Definition of the Weight Space}

The determination of the weight value is closely related to the expert's work experience, knowledge structure system and the degree of grasp of the evaluated object. Scientific and reasonable processing of the evaluation results of the expert group on the weight is an important link in the reasonable determination of the weight of each attribute.

Assuming that the $n$-dimensional of the weight vectors given by a finite group of different experts obey a certain distribution, the relevant parameter quantities of the distribution are determined according to the weight sample information that has been obtained, and then random weight samples that meet the requirements are drawn from the known weight distribution through the sampling method. In this way, the required weight sample data amount is obtained. The concept of the weight space is introduced. The weight space can be defined as a set of all possible weight combinations, which is expressed as:

$$
W=\left\{W_{k} \in R^{n} \mid w_{j k} \geq 0, \sum_{j=1}^{n} w_{j k}=1\right\}
$$

There, the value range of the weight is $0-100 \%$, and the sum of all weight is $100 \%$.

\subsection{Weight Space Constraints}

More weight vector data can be obtained for comprehensive evaluation by sampling the high-dimensional weight space of multiple attributes, and more reasonable comprehensive evaluation results can be obtained. Because the importance of each attribute is different from each other, two points need to be determined when sampling the weight space: (1) the constraint condition that the weight of each attribute satisfies; (2) the probability that the weight vector given by different weighting methods or expert groups is satisfactorily distributed. The constraints on the corresponding weight of each attribute of the weight vector include the weight interval of a single attribute, the relative relationship between the weight of two attributes, and so on. The constraints on the corresponding weight of each attribute of the weight vector include the weight interval of a single attribute, the relative relationship between the weight of two attributes, and so on. If the $i$-th attribute interval of a given weight vector $W_{k}$ is $w_{i k}\left[w_{i k \min }, w_{i k \max }\right]$, then the sum of all attribute weights is 1 , and the interval constraint can be converted to,

$$
\frac{w_{i k \min }}{1-w_{i k \min }} \leq \frac{w_{i k}}{\sum_{j \in[1, n]_{, j \neq 1}} w_{i k}} \leq \frac{w_{i k \max }}{1-w_{i k \max }}
$$

According to the above formula, the interval constraint of a single attribute can be transformed into a relative relation constraint with the sum of the weights of the remaining attributes. 
The relative relationship constraint between the $i$-th and $j$-th attribute weights of the weight vector $W_{k}$ is expressed as:

$$
\frac{w_{i k}}{w_{j k}} \geq \eta_{w_{i j}}
$$

where, $\eta_{w_{i j}}$ is a constrained weight space of order $n$. Taking the third-order weight vector as an example $\left(W_{K}=\left[w_{1 k}, w_{2 k}, w_{3 k}\right]^{\mathrm{T}}\right)$, the three-dimensional weight space constrained by two conditions in Figure $1\left(w_{1 k} \in[0.1,0.8], w_{1 k} / w_{3 k} \leq 3\right)$. The three-dimensional weight space is represented by the equilateral triangles. The area of the upper triangle of the parallel lines $L_{1}$ and $L_{2}$ represents the size of the weight $w_{1 k}$. The area of the lower trapezoid represents the sum of weights $w_{2 k}$ and $w_{3 k}$. The middle trapezoidal region surrounded by $L_{1}$ and $L_{2}$ is the feasible region of the weight space determined by the $w_{1 k}$ interval constraint. One end of $L_{3}$ is vertex $w_{2 k}$, and the ratio of the area of the upper and lower triangles is $w_{1 k} / w_{3 k}$. The feasible region formed by $L_{1}, L_{2}$, and $L_{3}$ is the constrained weight space.

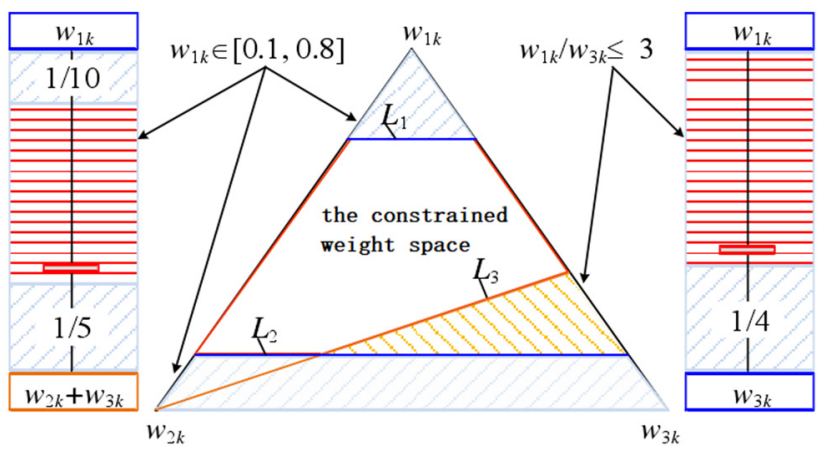

Figure 1. Constraints of high-dimensional weight space.

We can extract the weight vector of the known probability distribution from the constrained weight space, and perform multi-attribute comprehensive evaluation on the object to be evaluated, based on extracting a given number of weighted sample sets, and finally obtain the status result of each object to be evaluated.

\section{High-Voltage Cable Condition Evaluation Based on Markov Chain Monte Carlo (MCMC) Sampling in Weight Space}

\subsection{MCMC Sampling}

(1) Markov Chain

Markov Chain (Markov Chain, MC) is a discrete-time random process that satisfies Markov properties in mathematics. According to the transition probability between each state, we can transit from the current state to the rest of the state or transition to its own state. The mathematical definition of a Markov chain is [22]:

There, $\left\{x_{t}, t \in T\right\}$ is set to a random process. For any positive integer $n$ and $t_{1}<t_{2}<\ldots<t_{n}$, there will be:

$$
P\left\{X_{t_{1}}=x_{1}, \cdots, X_{t_{n-1}}=x_{n-1}\right\}>0
$$

and the conditional distribution is:

$$
\begin{gathered}
P\left\{X_{t_{n}} \leq x_{n} \mid X_{t_{1}}=x_{1}, \cdots, X_{t_{n-1}}=x_{n-1}\right\} \\
=P\left\{X_{t_{n}} \leq x_{n} \mid X_{t_{n-1}}=x_{n-1}\right\}
\end{gathered}
$$

$\left\{x_{t}, t \in T\right\}$ is called the Markov process. In the random process, the conditional probability can satisfy the following formula within any integer $n \in T$ and any $i_{0}, i_{1}, \ldots, i_{n+1} \in I$.

$$
\begin{aligned}
P\left\{X_{n+1}\right. & \left.=i_{n+1} \mid X_{0}=i_{0}, X_{1}=i_{1}, \cdots, X_{n}=i_{n}\right\} \\
& =P\left\{X_{n+1} \leq i_{n+1} \mid X_{n}=i_{n}\right\}
\end{aligned}
$$

$\left\{x_{n}, n \in T\right\}$ is called the Markov chain. 
In the Markov process, the matrix formed by the transition probabilities of each state is called the transition probability matrix, which is denoted as $P=\left[P_{i j}\right]_{n x n}$, where $P_{i j}$ represents the probability that the system is in state $i$ at time $t$ and state $j$ at time $t+1$. The state transition matrix $P$ and the probability distribution $\pi(x)$ satisfy the following expressions for all $i$ and $j$ :

$$
\pi(i) P(i, j)=\pi(j) P(j, i)
$$

where, $\pi(x)$ is the stationary distribution of the state transition matrix $P$.

\section{(2) Monte Carlo}

The Monte Carlo method (MC) is a numerical method based on random experiments and statistical calculations. The problem to be solved is abstracted as a mathematical problem and solved by establishing a probability model. The principle is to take the mathematical expectation of random variables or the probability of occurrence of random events as the solution to the problem to be sought.

First of all, a probabilistic process was constructed artificially, and some of its parameters are just the solutions to the problems to be sought. Essentially, the process is to transform non-random problems into random problems. Secondly, the constructed various probability models can be considered to be composed of different probability distributions, so simulation experiments can be realized by generating random vectors (variables) with known probability distributions. Finally, when the simulation experiment is completed, the solution of the problem is obtained based on the construction of various relevant estimators for the simulation experiment results that need to be investigated and registered.

\section{(3) MCMC sampling}

The Markov chains with Monte Carlo (Markov chains Monte Carlo, MCMC) sampling methods were combined to sample the high-dimensional weight space, and enough weighting schemes can be obtained. Gibbs sampling in MCMC is used to obtain a series of weight samples approximately equal to a given multi-dimensional probability distribution.

The weight space sampling based on MCMC mainly includes three steps:

(1) A suitable Markov chain is constructed in the weight space $W$, which can converge to a stable distribution $\pi(x)$;

(2) A sequence of sample points $x_{1}, x_{2}, x_{3}, \ldots x_{N}$ is generated based on the Markov chain in step 1), which starts from a certain point $x_{0}$ in $W$;

(3) When the Markov chain is iterated by $M$ samples that starts from $x_{0}$, the marginal probability distribution of $x_{M}$, at each moment is a stable distribution $\pi(x)$ and reaches convergence.

The distribution that the weight vector satisfies is unknown in the actual situation. It is necessary to estimate the random distribution that the weight vector of the expert group may satisfy based on the limited weight vector data. The $n$-dimensional weight vectors given by different expert groups obey the $n$-dimensional normal distribution supposed, which is $W \sim N_{n}\left(\mu, \sum\right)$, where $\mu$ is the expectation vector and $\sum$ is the covariance matrix. When $q$ elements in $W$ are unknown $(q<n)$ and other elements are known, there $W, \mu$, and $\sum$ can be divided into block matrices.

$$
W=\left[\begin{array}{l}
W^{(1)} \\
W^{(2)}
\end{array}\right], \mu=\left[\begin{array}{l}
\mu^{(1)} \\
\mu^{(2)}
\end{array}\right], \sum=\left[\begin{array}{ll}
\sum_{11} & \sum_{12} \\
\sum_{21} & \sum_{22}
\end{array}\right]
$$

where, $W^{(1)}$ and $\mu^{(1)}$ are the weight and expectation vector of $q \times 1$ order. $\sum_{11}$ is the $q \times q$ order covariance sub-matrix. The posterior conditional distribution of each element of different $n$-dimensional weight vectors at the given time of other elements can be obtained 
when the different elements form $W^{(1)}$ is chose and $q=1$. The conditional distribution of weight vector elements $w_{1}$ and $w_{2}$ can be obtained at $n=2$.

$$
\left\{\begin{array}{l}
w_{1} \sim N\left(\mu_{1}+\rho\left(w_{2}-\mu_{2}\right), 1-\rho^{2}\right) \\
w_{2} \sim N\left(\mu_{2}+\rho\left(w_{1}-\mu_{1}\right), 1-\rho^{2}\right)
\end{array}\right.
$$

The MCMC sampling output based on the software Matlab is shown in Figure 2. The Markov chain in MCMC sampling moves forward in the direction of $x_{1}$, and then moves in the direction of $x_{2}$ to perform alternate sampling of the coordinate axis, which can be seen from Figure 2 in each iteration.

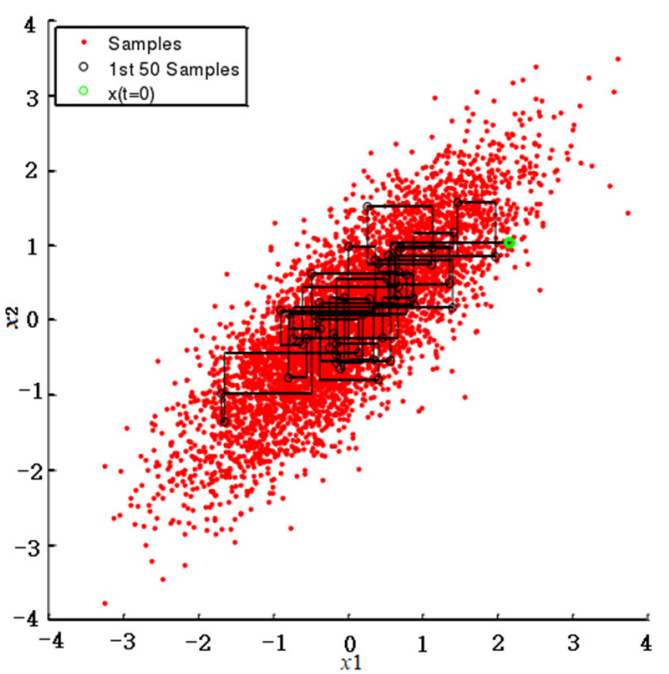

Figure 2. Distribution of Markov chain Monte Carlo (MCMC) sampling result.

\subsection{Comprehensive Evaluation Process}

The multi-attribute comprehensive evaluation problem contains $\mathrm{m}$ objects $S_{i}(i=1$, $2, \ldots, n)$ to be evaluated, and each object has $\mathrm{n}$ attributes. It can be expressed as $S_{i}=\left[x_{i 1}\right.$, $\left.x_{i 2}, \ldots, x_{i n}\right]$. Each object attribute should be standardized or degraded as $F\left(S_{i}\right)=\left[f\left(x_{i 1}\right)\right.$, $\left.f\left(x_{i 2}\right), \ldots, f\left(x_{i n}\right)\right]$ in multi-attribute decision-making to obtain a standardized data matrix $F(S)$ as shown in Table 1. The comprehensive degree of degradation is used as a measure of the final state of the cable, since the degree of degradation can characterize the health of power equipment. The comprehensive evaluation results of cables are divided into four intervals: $(0,0.25),(0.25,0.5),(0.5,0.75),(0.75,1)$, corresponding to the normal Q/GDW11262-2014 power cable and channel maintenance procedures status, attention status, abnormal status, and severe status.

Table 1. Original data table (deteriorative degree).

\begin{tabular}{ccccc}
\hline Evaluation Object & Attribute 1 & Attribute 2 & $\ldots$ & Attribute $\boldsymbol{n}$ \\
\hline$S_{1}$ & $f\left(x_{11}\right)$ & $f\left(x_{12}\right)$ & $\ldots$ & $f\left(x_{1 n}\right)$ \\
$S_{2}$ & $f\left(x_{21}\right)$ & $f\left(x_{22}\right)$ & $\ldots$ & $f\left(x_{2 n}\right)$ \\
$\ldots$ & $\ldots$ & $\ldots$ & $\ldots$ & $\ldots$ \\
$S_{m}$ & $f\left(x_{m 1}\right)$ & $f\left(x_{\mathrm{m} 2}\right)$ & $\ldots$ & $f\left(x_{m n}\right)$ \\
\hline
\end{tabular}

The weights of each attribute given by the expert group or the weights obtained by the weight space sampling method will be inconsistent in practical applications. There are $l$ types of empowerment schemes is supposed that expressed as $W_{k}=\left[w_{1 k}, w_{2 k}, \ldots, w_{n k}\right]^{\mathrm{T}}$, 
$k=1,2, \ldots, l$. The comprehensive evaluation result vector of each evaluated object under different weights can be described as:

$$
\left[\begin{array}{c}
V\left(S_{1}, W\right) \\
V\left(S_{2}, W\right) \\
\vdots \\
V\left(S_{m}, W\right)
\end{array}\right]=\left[\begin{array}{c}
F\left(S_{1}\right) \\
F\left(S_{2}\right) \\
\vdots \\
F\left(S_{m}\right)
\end{array}\right]\left[\begin{array}{cccc}
w_{11} & w_{12} & \cdots & w_{1 l} \\
w_{21} & w_{22} & \cdots & w_{2 l} \\
\vdots & \vdots & \ddots & \vdots \\
w_{n 1} & w_{n 2} & \cdots & w_{n l}
\end{array}\right]
$$

The probability matrix of $S_{i}$ belonging to different states can be obtained based on the $1 \times l$-order comprehensive evaluation result vector $V\left(S_{i}, W\right)$ of the evaluation object $S_{i}$ under $l$-weighting scheme, which can be expressed as follows:

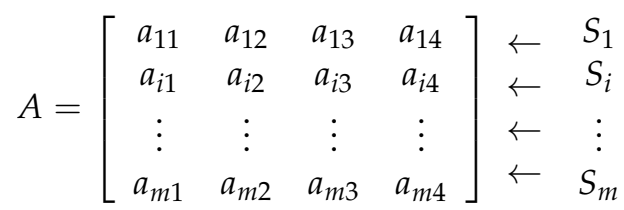

where, $a_{m 1}, a_{m 2}, a_{m 3}, a_{m 4}$ are representing channel maintenance procedures status, attention status, abnormal status, and severe status. For any two evaluated objects $S_{i 1}$ and $S_{i 2}$, the probability that the evaluation result of $S_{i 1}$ is better than $S_{i 2}$ under a given weight vector distribution is:

$$
P\left(S_{i 1} \geq S_{i 2}\right)=P\left\{V\left(S_{i 1}, W_{k}\right) \leq V\left(S_{i 2}, W_{k}\right)\right\}
$$

where, $P\left(S_{i 1}, S_{i 2}\right)$ is called the priority ranking probability of $S_{i 1}$ to $S_{i 2}$ under the set of weight vectors, and $W_{k}$ is the random variable in the weight space.

$V\left(S_{i}, W\right)$ is the $1 \times l$-order comprehensive evaluation result vector of the evaluated object $S_{i}$ under $l$-weighting scheme. $F\left(S_{i}\right)$ is the normalized data vector of $1 \times n$-order of the object $S_{i}$. $W_{k}$ is the $n \times 1$-order weight vector of the $k$-th weighting scheme.

Pairs of $m$ evaluated objects can be combined to get $m^{2}-m$ combinations. Each combination can be performed MCMC simulation by using constraint weight space. Finally, the priority probability $P$ between the two objects is calculated. MCMC simulation is performed on the combination of $m^{2}-m$ evaluated objects, and the priority ranking probability matrix of $m$ evaluated objects can be described as:

$$
P=\left[P_{i j}\right]_{m \times m}=\left[\begin{array}{cccc}
P_{11} & P_{12} & \vdots & P_{1 m} \\
P_{21} & P_{22} & \vdots & P_{2 m} \\
\ldots & \ldots & \ddots & \ldots \\
P_{m 1} & P_{m 2} & \vdots & P_{m m}
\end{array}\right] \text { For } \forall i, j
$$

where, $P$ is the $m \times m$-order priority ranking probability matrix, and the diagonal elements are $P_{m m}=0.5$. The off-diagonal elements are $P_{i j}=P\left(S_{i} \geq S_{j}\right)$, and there exist $P_{i j}+P_{j i}=1$ theoretically., there is $\left|P_{i j}+P_{j i}-1\right| \leq \alpha$ (the simulation error $\left.\alpha=0.001\right)$, and the precise values of $P_{i j}$ and $P_{j i}(i, j=1,2, \ldots, m)$ can be simulated independently, which shows that the credibility of the simulation results can be supported by the number of simulations, otherwise the number of simulations $l$ needs to be increased. According to the non-diagonal elements of each row of the priority ranking probability matrix $P$, the overall priority ranking probability $H_{i}$ of the $i$-th evaluated object can be described as:

$$
H_{i}=\frac{1}{m-1} \sum_{\substack{j=1 \\ j \neq i}}^{m} P_{i j}(i, j=1,2, \cdots, m)
$$


where $0 \leq H_{i} \leq 1$ it means that the evaluated object is definitely better than other objects when $H_{i}=1$, and the comprehensive evaluation result of this object ranks at the bottom of all objects when $H_{i}=0$. Assuming that the overall priority ranking probability vector of all evaluated objects is $H=\left(H_{1}, H_{2}, \ldots, H_{m}\right)$. It takes $H_{i}$ as the comprehensive evaluation result of the $i$-th evaluated object. The evaluated objects is prioritized according to the size of $H_{i}$. The maintenance sequence can be determined according to the sorting result is $S_{m}>S_{m-1}>\ldots>S_{1}$, when the sequence after sorting is $H_{1}>H_{2}>\ldots>H_{m-1}>H_{m}$. The reliability of sorting with $S_{m}$ is measured by the reliability coefficient $\theta$, and the calculation formula can be expressed as:

$$
\begin{gathered}
\theta\left(S_{1}, S_{2}, \cdots, S_{m}\right)={ }^{(m-1)} \sqrt{\prod_{i=1}^{m-1} \sigma_{i}} \\
\sigma_{i}=\sum_{k=i+1}^{m} P\left(S_{i}, S_{k}\right) /(m-i)
\end{gathered}
$$

The $i$ represents the average priority ranking probability of the evaluation object $S_{i}$ relative to the subsequent evaluation object in the $i$-th place. In summary, the specific steps for comprehensive evaluation of high-voltage cable status are as follows:

(1) A single abnormality judgment is performed on the index with high priority based on the collected cable sample data. These cables that show obvious abnormal data in a single index are directly included in the scope of maintenance and defect identification. The remaining cable samples are comprehensively evaluated.

(2) The degree of degradation of the cable data to be evaluated is obtained based on the above formula.

(3) There $l$-weight vector samples (given by the relevant decision maker) of the highdimensional weight space are inferred by MCMC sampling. The probability matrix $A$ and priority ranking probability matrix $P$ of each evaluation object belonging to different states are obtained after weighted summation of the sample degradation degree and pairwise comparison.

(4) The overall priority ranking probability $H_{i}$ and the credibility coefficient $\sigma_{i}$ of the $i$-th evaluated object are obtained based on the priority ranking probability matrix. Finally, the inspection scope and the priority of the inspection are determined according to the sorting results.

\section{Comprehensive State Evaluation of High-Voltage Cables}

The data sources for comprehensive state evaluation and defect identification of highvoltage cables can be divided into five aspects: online monitoring data, offline test data, operation and maintenance information, equipment information and environmental and external force damage data, which is shown in Figure 3. According to the actual situation of $220 \mathrm{kV}$ high-voltage cables in a certain power supply bureau, this paper verifies the highvoltage cable condition evaluation method proposed based on the multi-source information of high-voltage cables.

The partial discharge ultrasonic sensor and grounding circulation sensor are mainly installed on the grounding wire of each terminal and intermediate joint of the cable, and the cable temperature data is measured through distributed optical fibers. There are differences of monitoring and test data in the sampling period, data structure, measurement location, etc. For example, each cable usually has multiple cable terminals and intermediate joints with the grounding circulation and partial discharge data of cables. Multiple ground circulation and partial discharge monitoring data are received at every acquisition time. The monitoring data of the entire line length can be obtained at each acquisition moment for the temperature and current carrying capacity of high-voltage cables. It constitutes a data set of the cable real-time data based on online monitoring and other offline data. 


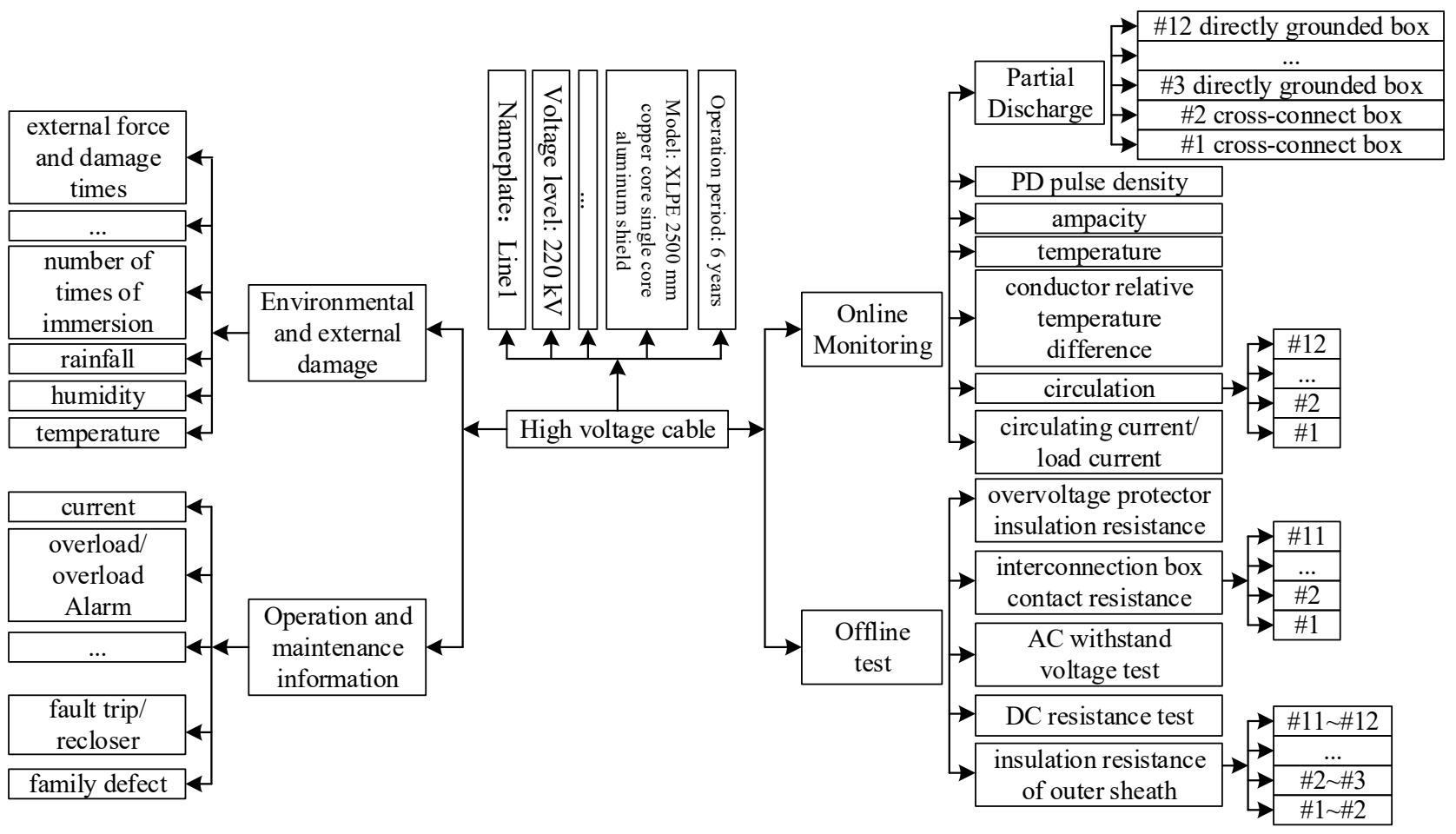

Figure 3. The data source of status for high-voltage cable.

\subsection{Selection of Comprehensive Evaluation Indexes for High-Voltage Cable State}

The health of the cables will gradually change during the operation of high-voltage cables, due to factors such as operating time and working environment. In order to ensure the comprehensive evaluation result of the high-voltage cable status is accurate, the factors that have a certain impact on the cable operating status should be selected as comprehensively as possible. The comprehensive evaluation indicators of cable status selected in this paper are shown in Table 2.

Table 2. The state comprehensive evaluation index of high-voltage cable.

\begin{tabular}{cccc}
\hline Index & Label & Index & Label \\
\hline Cable temperature & $X_{1}$ & Overhaul times & $X_{12}$ \\
Ground circulation & $X_{2}$ & Number of failures & $X_{13}$ \\
Partial Discharge & $X_{3}$ & Family defect & $X_{14}$ \\
Ampacity & $X_{4}$ & Ambient temperature & $X_{15}$ \\
AC withstand voltage & $X_{5}$ & Environment humidity & $X_{16}$ \\
Outer sheath insulation resistance & $X_{6}$ & Rainfall & $X_{17}$ \\
Grounding resistance of interconnection box & $X_{7}$ & Number of flooding & $X_{18}$ \\
Insulation resistance of overvoltage & $X_{8}$ & Number of ant infestations & $X_{19}$ \\
protector & $X_{9}$ & Whether the channel is sinking & $X_{20}$ \\
Load current & $X_{10}$ & Number of external damage & $X_{21}$ \\
Overload/overload alarm & $X_{11}$ & & \\
Fault trip/reclose & & & \\
\hline
\end{tabular}

When the degradation degree is set to 0 , it indicates that the device is in a healthy state. when the degradation degree is set to a value of 1 , it indicates that the device is in a fault state. The final state of the cable is measured by the comprehensive degradation degree in this paper. It is generally hoped that the number of failures or overhauls is as small as possible for discrete data such as the number of failures and the number of overhauls. The calculation formula for defining their degradation degree is as follows:

$$
g=\left\{\begin{array}{c}
0, X<X_{\min } \\
1-\frac{X_{\min }}{X}, X \geq X_{\min }
\end{array}\right.
$$


where, $X$ and $X_{\min }$ are the historical faults or overhaul times since the cable was put into operation and the corresponding limit of times.

\subsection{Case Analysis}

There are 20 circuits of $220 \mathrm{kV}$ cables in the jurisdiction of a power supply bureau, of which 12 circuits are equipped with optical fiber temperature monitoring devices, and 16 are equipped with cable outer sheath circulation monitoring devices; there are also 7 cables with partial discharge monitoring devices installed. A total of 15 cables with five cycles of monitoring devices for temperature, partial discharge, and grounding circulation are installed at the same time as examples to test the effectiveness of the comprehensive evaluation method in this paper. The basic information of each evaluated cable is shown in Table 3. The cable model is $220 \mathrm{kV}-\mathrm{XLPE}-2500 \mathrm{~mm}$ copper core single core aluminum sheath. The worst value of cable online monitoring, offline test, operation and maintenance and environmental historical data before 20 July 2020 was chosen to comprehensively evaluate the operating status of each cable, assuming that all collected data correspond to the true status of the cable.

Table 3. Basic information of cables.

\begin{tabular}{|c|c|c|c|c|c|c|c|c|c|}
\hline $\begin{array}{l}\text { Line } \\
\text { Number }\end{array}$ & $\begin{array}{l}\text { Number of } \\
\text { Segments }\end{array}$ & $\begin{array}{c}\text { Number of } \\
\text { Cables }\end{array}$ & $\begin{array}{l}\text { Putting into } \\
\text { Operation Time }\end{array}$ & $\begin{array}{l}\text { Length } \\
\text { (m) }\end{array}$ & $\begin{array}{l}\text { Number of } \\
\text { Terminals }\end{array}$ & $\begin{array}{l}\text { Number of } \\
\text { Middle } \\
\text { Joints }\end{array}$ & $\begin{array}{c}\text { Number of } \\
\text { Over- } \\
\text { Voltage } \\
\text { Protectors }\end{array}$ & $\begin{array}{l}\text { Number of } \\
\text { Cross- } \\
\text { Connected } \\
\text { Boxes }\end{array}$ & $\begin{array}{c}\text { Maximum } \\
\text { Current } \\
\text { Carrying } \\
\text { Capacity (A) }\end{array}$ \\
\hline$S_{1 A}$ & \multirow{3}{*}{12} & \multirow{3}{*}{3} & \multirow{3}{*}{15 June 2013} & \multirow{3}{*}{6550} & \multirow{3}{*}{3} & \multirow{3}{*}{36} & \multirow{3}{*}{27} & \multirow{3}{*}{8} & \multirow{3}{*}{880} \\
\hline$S_{1 B}$ & & & & & & & & & \\
\hline$S_{1 C}$ & & & & & & & & & \\
\hline$S_{2 A}, S_{2 B}$ & \multirow{2}{*}{12} & \multirow{2}{*}{3} & \multirow{2}{*}{15 June 2013} & \multirow{2}{*}{6550} & \multirow{2}{*}{3} & \multirow{2}{*}{36} & \multirow{2}{*}{27} & \multirow[t]{2}{*}{8} & \multirow{2}{*}{880} \\
\hline$S_{2 C}$ & & & & & & & & & \\
\hline$S_{3 A}$ & \multirow{3}{*}{12} & \multirow{3}{*}{3} & \multirow{3}{*}{15 August 2016} & \multirow{3}{*}{6550} & \multirow{3}{*}{3} & \multirow{3}{*}{36} & \multirow{3}{*}{27} & \multirow{3}{*}{8} & \multirow{3}{*}{880} \\
\hline$S_{3 B}$ & & & & & & & & & \\
\hline$S_{3 C}$ & & & & & & & & & \\
\hline$S_{4 A}$ & \multirow{3}{*}{21} & \multirow{3}{*}{3} & \multirow{3}{*}{15 September 2014} & \multirow{3}{*}{10,500} & \multirow{3}{*}{6} & \multirow{3}{*}{60} & \multirow{3}{*}{42} & \multirow{3}{*}{14} & \multirow{3}{*}{2100} \\
\hline$S_{4 B}$ & & & & & & & & & \\
\hline$S_{4 C}$ & & & & & & & & & \\
\hline$S_{5 A}$ & \multirow{3}{*}{21} & & & & & & & & \\
\hline$S_{5 B}$ & & 3 & 15 September 2014 & 10,500 & 6 & 60 & 42 & 14 & 2100 \\
\hline$S_{5 C}$ & & & & & & & & & \\
\hline
\end{tabular}

Firstly, whether each cable has a single indicator abnormal state need to be judged. It can be seen that the conductor temperature of Phase A near the GIS terminal of the substation $750 \mathrm{~m}$ exceeds the maximum allowable temperature of $130{ }^{\circ} \mathrm{C}$ based on the temperature data of Line 1 in Figure 4, and it is judged as $S_{1 A}$ abnormal. According to the ratio of the circulating current to the load current at each terminal and intermediate joint of Line 2 in Figure 5, it can be seen that the circulating current at the B-phase directly grounded joint \#9 exceeds $20 \%$ of the load current, and it is judged that the cable $S_{2 B}$ is abnormal.

Where, \#0 represents the GIS terminal of the substation. There \#1,2,4,5,7,8,10,11 represent the cross interconnection connector of cable. There \#3,6,9,12 represent the directly grounded connector of cable.

The insulation resistance value of the outer sheath of the abnormal section of Line 3 and Line 4 in Table 4 . It can be seen from the table that the insulation resistance of the outer sheath of each phase in the \#4-\#5 sections of Line 3 does not meet the requirement of less than $0.5 \mathrm{M} \Omega / \mathrm{km}$. The insulation resistance of the outer sheath of the \#9-\#10 sections and $\# 12-\# 13$ sections of Line 4 are also unqualified, so it is determined that the cables $S_{3 A}, S_{3 B}$, $S_{3 C}$ and $S_{4 A}, S_{4 B}, S_{4 C}$ are abnormal. 


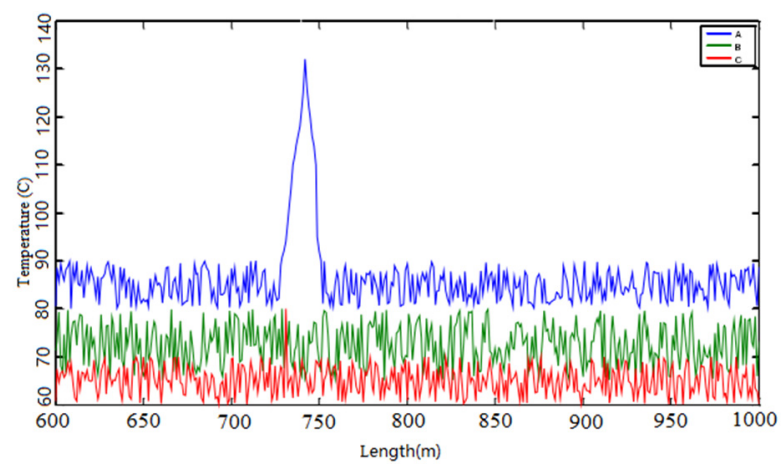

Figure 4. Temperature data of line 1.

- $\circ \mathrm{B} \bullet \mathrm{C}$

Percentage of circulating current and load current of Line 2

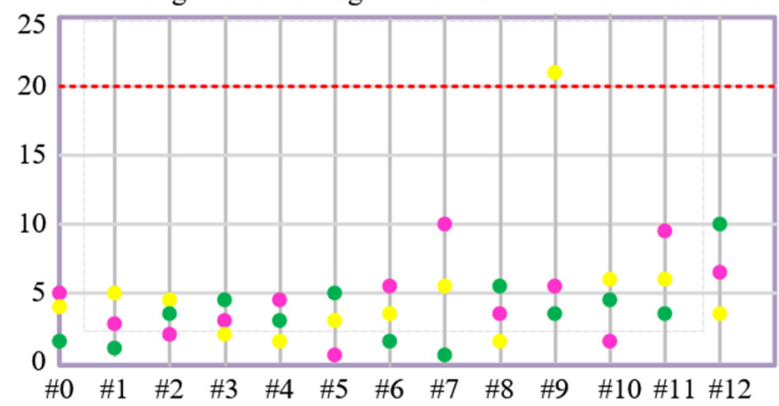

Figure 5. The ratio of the loop current and load current of line 2.

Table 4. Insulation resistance value of outer sheath

\begin{tabular}{cccccc}
\hline \multirow{2}{*}{ Line Number } & \multirow{2}{*}{ Part } & \multicolumn{3}{c}{ Insulation Resistance (M) } & \multirow{2}{*}{ Joint Well } \\
\cline { 3 - 6 } & & A & B & C & \\
\hline Line 3 & $\# 4-\# 5$ & 0 & 0 & 1.5 & $\# 4$ \\
\hline \multirow{2}{*}{ Line 4 } & $\# 9-\# 10$ & 0 & 0 & 1 & $\# 9$ \\
\cline { 2 - 5 } & $\# 12-\# 13$ & 0 & 10 & 0 & $\# 12$ \\
\hline
\end{tabular}

It can be determined that the eight cable lines $S_{1 A}, S_{2 B}, S_{3}$ and $S_{4}$ all have abnormalities in different situations, which is judged by a single index, and corresponding maintenance and repair work need to be prioritized. The abnormal cables are eliminated, and the validity of the evaluation method in this paper is tested based on the remaining seven cables. The test result is compared with the imported reference sample, which corresponds to the best value of each status indicator in the historical data set.

Actually, the distribution that the weight vector satisfies is not known, so it is necessary to estimate the random distribution that the weight vector of the expert group may satisfy based on the limited weight vector data. According to the estimated probability distribution, by constructing the transition conditional probability, enough weight sample data are drawn upon. The transition conditional probability is constructed according to the estimated probability distribution, and extract enough weighted sample data. The amount of available weight sample data is artificially expanded based on this method. Since the uncertainty and difference of the weights are considered, the evaluation results are more reasonable. This is assuming that the weight vectors given by multiple experts satisfy the n-dimensional normal distribution.

The importance and reliability of cable data from different sources are different. The data obtained through offline testing are the most accurate, and the monitoring data may have errors in the monitored data due to sensor or communication abnormalities, so its accuracy is lower than offline testing. In practice, cable failures occur from time to time due 
to external force damage such as construction, but the probability of occurrence is lower than the accuracy of offline testing and online monitoring data. The environment has the weakest influence on the evaluation of cable status. Therefore, the reliability of the cable status data in Table 2 from high to low is offline test data, online monitoring data, external force damage data, operation and maintenance data, and environmental data. The relative relationship of the weight vectors of various indicators can be obtained as:

$$
\begin{aligned}
& \left\{w_{5}, w_{6}, w_{7}, w_{8}\right\} \geq\left\{w_{1}, w_{2}, w_{3}, w_{4}\right\} \geq\left\{w_{18}, w_{19}, w_{20}, w_{21}\right\} \\
& \geq\left\{w_{9}, w_{10}, w_{11}, w_{12}, w_{13}, w_{14}\right\} \geq\left\{w_{15}, w_{16}, w_{17}\right\}
\end{aligned}
$$

where, $w_{5}>\left(w_{1}, w_{2}, w_{3}, w_{4}\right)$ is equivalent to $w_{5} / w_{1}>1, w_{5} / w_{2}>1, w_{5} / w_{3}>1$ and $w_{5} / w_{4}>1$. By analogy, 74 conditional constraints of the weight vector can be obtained. Therefore, the 500 weight vector obtained by MCMC sampling for the constrained weight space is shown in Figure 6.

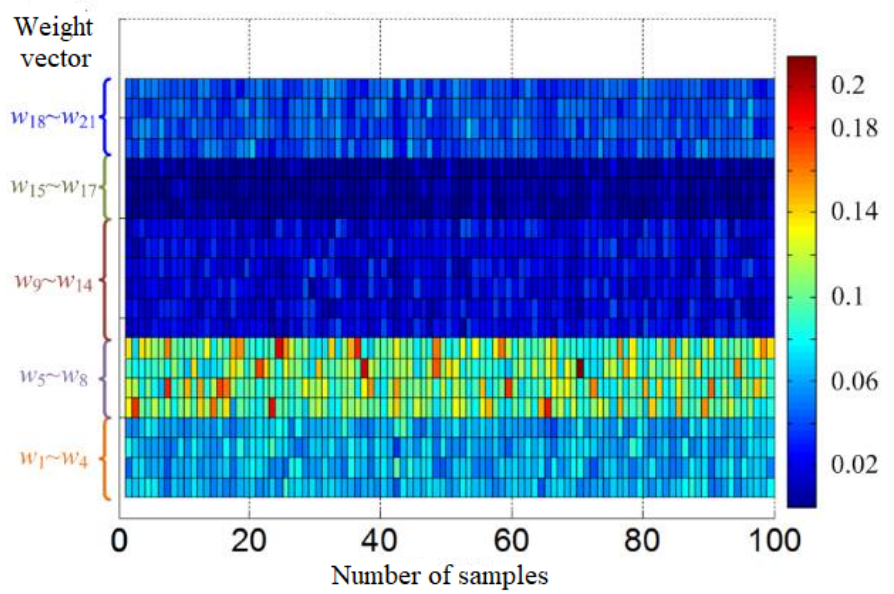

(a)

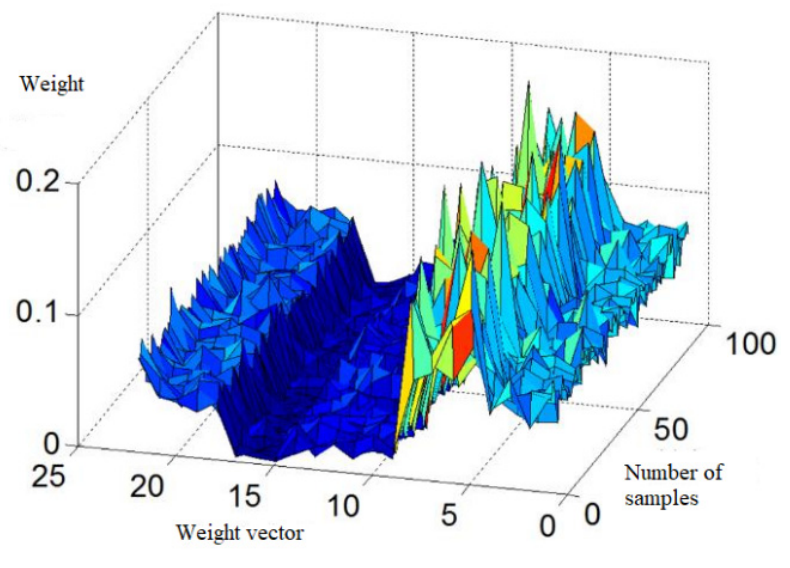

(b)

Figure 6. Sampling results of weight space by MCMC. (a) Plane projection; (b) three-dimensional map.

The sampling results of the first 100 sets of weight vectors are shown in Figure 5, where the maximum weight is distributed in the interval $w_{5}-w_{8}$, and the maximum value does not exceed 0.2. The weights of cable operation and maintenance and environmental data $w_{9}-w_{14}$ and $w_{15}-w_{17}$ are relatively small. The operating status of the cable is mainly reflected by offline testing, online monitoring and external force damage data, but the weights of different status indicators show random distribution characteristics under given constraints. According to the weight vector sampling results in Figure 5 and the evaluated cable data of the reference sample, the evaluation results and their probability distributions of seven evaluated cables can be obtained, as shown in Figures 7 and 8.

The probability of the cable $S_{1 B}$ is 1 , which has priority over $S_{1 C}, S_{2 A}, S_{2 C}$, and $S_{5 C}$, and the probability of priority over $S_{5 A}(0.994)$ and $S_{5 B}(0.812)$, there the cable $S_{1 B}$ is in the best state of all the cables evaluated. It can be seen from Figure 6 that the evaluation results of $S_{1 B}$ are relatively smallest under most of the weight vectors, but in the 500 groups of weight vectors, there are still 94 groups and 3 groups of weight vectors. The evaluation results of $S_{1 B}$ are worse than $S_{5 A}$ and $S_{5 B}$. According to Formula (12), the overall priority ranking probability vector can be calculated as $H=[0.8295,0.44$, $0.0065,0.1396,0.5670,0.737,0.2915]^{\mathrm{T}}$, from which the order of the evaluated cable status is $S_{1 B}>S_{5 B}>S_{5 A}>S_{1 C}>S_{5 C}>S_{2 C}>S_{2 A}$. According to Formula (15), the credibility coefficient of this ranking is calculated as $\theta=0.981$. 


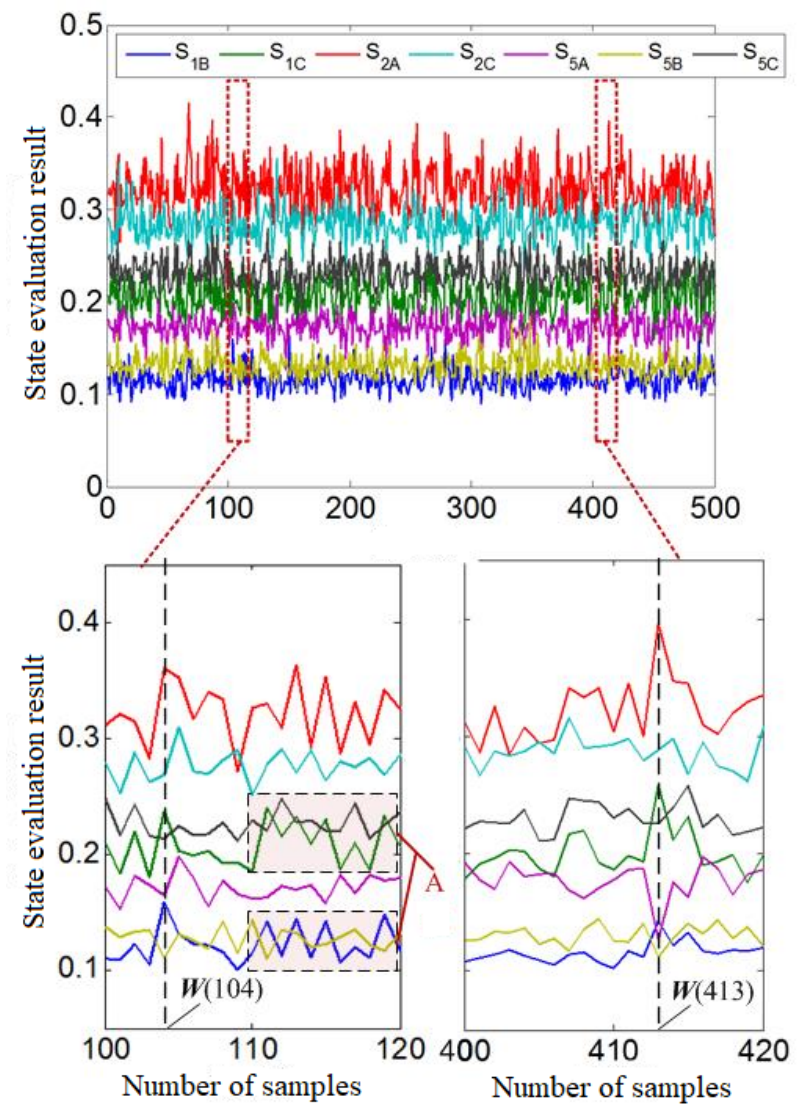

Figure 7. State evaluation results of cables.

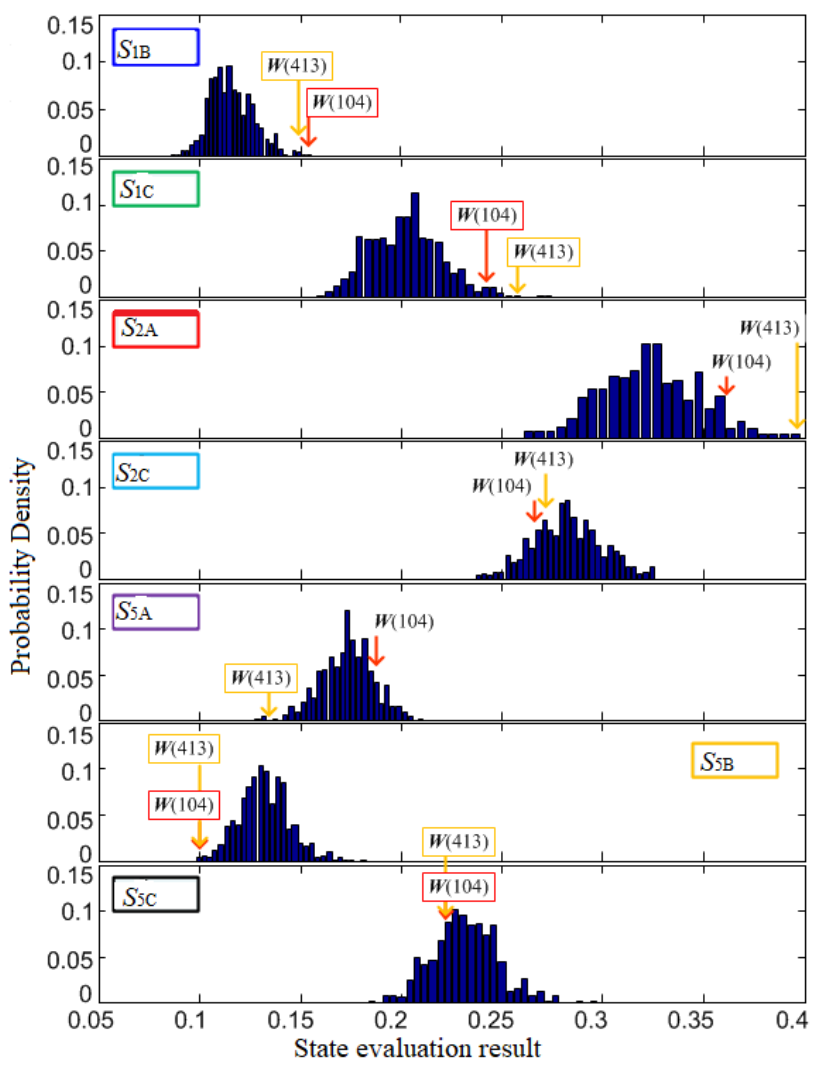

Figure 8. Probability distribution of state evaluation results of cables. 
According to the probability distribution of the status evaluation results of each cable in Figure 7, it can be seen that the relative relationship between the expected values of the evaluation results of different cables is consistent with the aforementioned status ranking results. The evaluation results of $S_{1 B}, S_{5 B}$, and $S_{5 A}$ are distributed in the overlapping area; the evaluation results of $S_{1 C}$ and $S_{2 A}$ have a large distribution interval, which shows that the dispersion of the weight vector has a great influence on the evaluation of the state of $S_{1 C}$ and $S_{2 A}$. When MCMC is not used to sample the weight space, the weight vector obtained is a fixed value. If the weight vectors obtained at this time are $W(104)$ and $W(413)$ as shown in Figure 7, the sorting results of each cable status are respectively $S_{5 B}>S_{1 B}>S_{5 A}>S_{5 C}>S_{1 C}>S_{2 C}>S_{2 A}$ and $S_{5 B}>S_{5 A}>S_{1 B}>S_{5 C}>S_{1 C}>S_{2 C}>S_{2 A}$, and the evaluation results of the corresponding cables are also marked in Figure 7.

The method in this paper performs MCMC sampling on the weight space, which can obtain the overall probability distribution of the relative relationship of the evaluated objects from the perspective of the randomness of the group weighting; compared with the existing weighting methods, the method in this paper is helpful for analyzing the weights in a given interval. The impact of internal changes on the evaluation results is shown.

According to Formula (11), the probability matrix of each evaluation cable belonging to different states is:

$$
\begin{array}{ll}
S_{1 B} & \rightarrow \\
S_{1 C} & \rightarrow \\
S_{2 A} & \rightarrow \\
S_{2 C} & \rightarrow \\
S_{5 A} & \rightarrow \\
S_{5 B} & \rightarrow \\
S_{5 C} & \rightarrow
\end{array}\left[\begin{array}{cccc}
1 & 0 & 0 & 0 \\
0.977 & 0.023 & 0 & 0 \\
0 & 1 & 0 & 0 \\
0.025 & 0.975 & 0 & 0 \\
1 & 0 & 0 & 0 \\
1 & 0 & 0 & 0 \\
0.858 & 0.143 & 0 & 0
\end{array}\right]
$$

Combining Figure 7 and the calculation results of the probability matrix, it can be seen that $S_{1 B}, S_{5 A}$, and $S_{5 B}$ are in the normal state, and $S_{1 C}$ and $S_{5 C}$ are in the normal state with a probability of $97.7 \%$ and $85.8 \%$, respectively. $S_{2 A}$ is in the interval of the attention state, and $S_{2 C}$ belongs to the attention state with a probability of $97.5 \%$. It can be seen that the temperature values of $S_{2 A}$ and $S_{2 C}$ exceed the allowable long-term operation temperature of $90{ }^{\circ} \mathrm{C}$ but do not exceed the maximum allowable temperature of $130{ }^{\circ} \mathrm{C}$ based on the cable data, and the amount of partial discharge is close to the extreme value of the normal aging standard. In practice, cable failure events rarely occur, and it can be seen that the result is consistent with the actual situation. In actual situations, cable failure events occur rarely, and it can be seen that the result is consistent with the actual situation. For this reason, it can be determined that $S_{2 A}$ and $S_{2 C}$ cables need to be prioritized for routine testing and periodic maintenance.

\section{Conclusions}

The weight vector set obtained by MCMC sampling proposed in this paper takes into account the difference and uncertainty of the weight, and can reflect the influence of the weight value on the evaluation result of the high-voltage cable state when the weight value changes within a given interval. The overall priority probability of each cable operating state can be obtained by constructing the priority sequence probability matrix of the evaluated cables, which can guide the operation and maintenance personnel to formulate the sequence of cable maintenance. The superiority of this method is even more obvious when hundreds or thousands of cables are being overhauled at the same time. The scope of maintenance can be shortened, and the cable lines to be inspected can be clarified, which greatly saves manpower and financial resources. 


\begin{abstract}
Author Contributions: Conceptualization, X.-K.M. and Y.-B.J.; methodology, Z.-H.L. and Z.-Q.Y.; validation, P.-J.H.; formal analysis, Z.-M.L.; investigation, T.J.; resources, X.-K.M.; data curation, Z.-H.L.; writing — original draft preparation, Z.-Q.Y.; writing—review and editing, Y.-B.J.; funding acquisition, X.-K.M. and Z.-H.L. All authors have read and agreed to the published version of the manuscript.

Funding: This work was supported in part by State Grid Shanxi Electric Power Corporation headquarter's science and technology project (52053020000V), the Hebei University “One Province One University" Special Fund (801260201232)", and the president fund project of Hebei University (XZJJ201908), and postdoctoral programme of State Grid Shanxi Electric Power Corporation and State Grid Shanxi Electric Power Corporation headquarter's.
\end{abstract}

Institutional Review Board Statement: Not applicable.

Informed Consent Statement: Not applicable.

Data Availability Statement: Not applicable.

Conflicts of Interest: The authors declare no conflict of interest.

\title{
References
}

1. Jian, Y.; Zhong, T. Design of online travelling wave based fault location algorithm for HV power cable. Power Syst. Prot. Control 2016, 44, 48-54.

2. Zhang, Y.-Y.; Jiang, F.-Y.; Yu, X.-Y.; Zhou, K.; Zhang, W.; Fu, Q.; Hao, J. Assessment of Thermal Aging Degree of 10kV Cross-Linked Polyethylene Cable Based on Depolarization Current. IEEE Access 2021, 9, 111020-111029. [CrossRef]

3. Wang, Z.-Y. Operation Condition Evaluation for XLPE Power Cables Based on Improved Radar Chart Theory. High Volt. Appar. 2015, 51, 145-150.

4. Yang, Y.; You, J.; Jia, Z.; Zhang, Y. Evaluation Analysis of 10 kV XLPE Cable's Operation Condition. High Volt. Eng. 2017, 43, 298-306.

5. Jorgens, C.; Clemens, M. A Review about the Modeling and Simulation of Electro-Quasistatic Fields in HVDC Cable Systems. Energies 2020, 13, 5189. [CrossRef]

6. $\quad$ Spack-Leigsnering, Y.; Ruppert, G.; Gjonaj, E.; De Gersem, H.; Koch, M. Towards Electrothermal Optimization of a HVDC Cable Joint Based on Field Simulation. Energies 2021, 14, 2848. [CrossRef]

7. Liang, Z.-B.; Xu, M.-Z.; Hou, J.-P.; Wang, L.; Zhai, H.-Q. Evaluation Method for Thermal State of Extra-high Voltage XLPE Power Cable Termination. High Volt. Eng. 2017, 43, 682-688.

8. Darco, S.; Suul, J.A.; Beerten, J. Configuration and Model Order Selection of Frequency-Dependent Models for Representing DC Cables in Small-Signal Eigenvalue Analysis of HVDC Transmission Systems. IEEE J. Emerg. Sel. Top. Inpower Electron. 2021, 9 , 2410-2426. [CrossRef]

9. Mazzanti, G.; Diban, B. The Effects of Transient Overvoltages on the Reliability of HVDC Extruded Cables. Part1: Long Temporary Over voltages. IEEE Trans. Power Deliv. 2021, 36, 3784-3795. [CrossRef]

10. Semenov, D.; Sidorova, A.; Romanov, P.; Kuvshinov, A. Examination of State of the Cable Insulation by the Return Voltage. Int. J. Emerg. Electr. Power Syst. 2018, 1-10. [CrossRef]

11. Asif, M.; Lee, H.Y.; Park, K.H.; Lee, B.W. Accurate Evaluation of Steady-State Sheath Voltage and Current in HVDC Cable Using Electromagnetic Transient Simulation. Energies 2019, 12, 4161. [CrossRef]

12. Dai, X.; Hao, J.; Jian, Z.; Liao, R.; Zheng, X.; Zhang, Y. High-voltage frequency domain spectroscopy analysis of a thermally aged XLPE submarine cable under continuous and cyclic voltage based on carrier transport and polarisation characteristics. High Volt. 2021. [CrossRef]

13. Dai, X.-Z.; Jian, H.; Liao, R.-J.; Zheng, X.; Gao, Z.; Peng, W. Multi-dimensional Analysis and Correlation Mechanism of Thermal Degradation Characteristics of XLPE Insulation for Extra High Voltage Submarine Cable. IEEE Trans. Dielectr. Electr. Insul. 2021, 28, 1488-1496. [CrossRef]

14. Meng, F.-B.; Chen, X.-R.; Dai, C.; Zhang, M.; Paramane, A.; Zheng, L.; Tanaka, Y. Effect of Thermal Ageing on Physico-Chemical and Electrical Properties of EHVDC XLPE Cable Insulation. IEEE Trans. Dielectr. Electr. Insul. 2021, 28, 1012-1019. [CrossRef]

15. Dai, X.-Z.; Hao, J.; Jian, Z.; Gao, Z.; Cheng, J.; Zheng, X. Ageing state identification and analysis of AC 500 kV XLPE submarine cable based on high-voltage frequency dielectric response. IET Sci. Meas. Technol. 2020, 14, 977-984. [CrossRef]

16. Bian, H.-R.; Yang, L.-J.; Qin, H.; Zhang, J.; Ma, Z.; Deng, B.; Zhang, H. Analysis of Free Energy Increment for Insulation Aging in Crosslinked Polyethylene DC Cable. IEEE Access 2019, 7, 77419-77425. [CrossRef]

17. Q/GDW11262-2014; State Grid Corporation of China Maintenance specification for power cables and channels. China Electric Power Press: Beijing, China, 2014.

18. Chen, Y.-D.; Zhou, K.; Kong, J.-M.; Akram, S.; Ren, X.; Zhang, X.; Li, Y.; Zhao, Q. Hydrogen evolution and electromigration in the corrosion of aluminium metal sheath inside high-voltage cables. High Volt. 2021. [CrossRef] 
19. Meng, F.-B.; Chen, X.-R.; Dai, C.; Zhang, M.; Shi, Y.; Paramane, A.; Muhammad, A. Interfacial microstructure and insulation properties of $500 \mathrm{kV}$ EHVDC XLPE cable factory joint under different roughness and degassing durations. Polym. Degrad. Stab. 2021, 192, 109688. [CrossRef]

20. Wang, P.Y.; Liu, G.; Ma, H.; Liu, Y.; Xu, T. Investigation of the Ampacity of a Prefabricated Straight-Through Joint of High Voltage Cable. Energies 2017, 10, 2050. [CrossRef]

21. Liu, Z.-H.; Liu, X.-L.; Zhang, Z.-Y.; Zhang, W.; Yao, J. Research on Optical Fiber Sensor Localization Based on the Partial Discharge Ultrasonic Characteristics in Long-Distance XLPE Cables. IEEE Access 2020, 8, 184744-184751. [CrossRef]

22. Roy, V. Convergence Diagnostics for Markov Chain Monte Carlo. Annu. Rev. Stat. Appl. 2020, 7, 387-412. [CrossRef]

23. Nemeth, C.; Fearnhead, P. Stochastic Gradient Markov Chain Monte Carlo. J. Am. Stat. Assoc. 2021, 116, 433-450. [CrossRef]

24. Hu, G.-Y.; Wang, H.-Y. Most Likely Optimal Subsampled Markov Chain Monte Carlo. J. Syst. Sci. Complex. 2021, 34, 1121-1134. [CrossRef] 